\title{
ADDITIVE LOGISTIC REGRESSION APPLIED TO RETINA MODELLING
}

\author{
Sérgio F. Martins, Leonel A. Sousa \\ IST/INESC-ID \\ Universidade Técnica de Lisboa \\ Lisboa, Portugal \\ $\{$ sfam,las $\} @$ sips.inesc-id.pt
}

\author{
João C. Martins \\ ESTIG/INESC-ID \\ Instituto Politécnico de Beja \\ Beja, Portugal \\ jcsm@sips.inesc-id.pt
}

\begin{abstract}
The accurate modelling of the human visual system, particularly of the retina, would be a great achievement and a big step in the development of visual prostheses. Several methods and algorithms have been proposed to accomplish such a difficult task, mainly to what concerns the adaptation and nonlinear mechanisms of the retina.

This paper presents the results obtained by the employment of additive logistic regression techniques to model the nonlinear block of a canonical Linear-Nonlinear-Poisson retina model, considering the spike triggering process from a statistical point of view, complemented with the PCA of the stimuli covariance matrix. The displayed results were obtained by modelling real retina data using different forms for the nonlinear block and are assessed with different error measures.
\end{abstract}

Index Terms - Retina Model; Spike-Triggered Analysis; Nonlinear Functionals; Additive Models

\section{INTRODUCTION}

The human visual system has been a challenging matter of study among researchers. Many different approaches have been used towards retina modelling, mainly adopting spiketriggered analysis [1], information-theoretic approach [2] and maximum-likelihood estimation [3].

The retina is composed by several cells layers, with intrinsic connections between different layers [4], that can be individually modeled. A common retina model architecture that has became very popular among researchers and is already considered a state-of-the-art model, is the Linear-NonLinearPoisson (LNP) model depicted in fig. 1, that models the retina as a whole.

By applying spike-triggered analysis, it is possible to extract several linear components from the stimuli that are relevant for the retina. Spike-triggered average and spike-triggered covariance combined with principal component analysis, has proven to be quite efficient for that purpose [5]. The Poisson block in the LNP model is responsible for generating spikes,

This work was partially supported by the Portuguese Foundation for Science and Technology (FCT) through Program FEDER and through the project RNC - Retina Neural Code: Accurate Modelling towards an Artifcial Visual System. given a time-varying probability of a spike being fired, known as firing rate. The most challenging part of this model is its nonlinear characteristics. The idea behind it is that the probability of a spike being elicited is a nonlinear functional of the linear components of the stimuli. The methods proposed so far to model the nonlinearity consists in estimating the firing rate from a set of trials of a single experiment, and then fit a nonlinear function if there is only one linear component [1]. For more than one linear component the most common method is to compute the ratio between the histogram of the input subset that elicited a spike and the input histogram, defining a multi-dimensional functional [5]. The problem is that to populate sufficiently a n-dimensional histogram, the amount of data needed grows exponentially with the number of linear kernels.

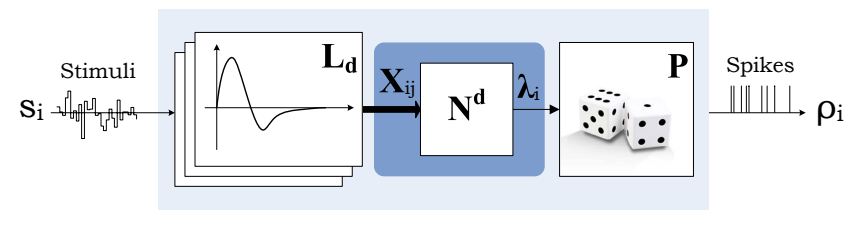

Fig. 1. Linear-NonLinear-Poisson (LNP) Model

To overcome this difficulty, we propose in this paper to use Generalized Additive Models to estimate the nonlinear functional. The firing rate can be interpreted as a probability, and for that reason, logistic regression methods are suitable to model it. However, since the retina shows highly nonlinear features in its response, the use of a more general form of this regression is proposed: the additive logistic regression. Its use is valid if the spike trains are sampled with a small time period so that there is, at most, one spike per time bin whose presence, or not, can be represented by a Bernoulli random variable. Experimental results show that the use of additive logistic regression allows to properly model the nonlinearity in the LNP like retina models.

\section{GENERALIZED ADDITIVE MODELS}

In the retina response, the output spike train $\rho$ can be seen as a sequence of events of two classes, where at time instant 
$i$ it can take one of two values, $\rho_{i}=1$ if a spike is triggered or $\rho_{i}=0$ otherwise. Statistically speaking, we wish to model the probability of a spike occurring $\mathrm{P}\left(\rho_{i}=1 \mid \mathbf{X}_{i j}\right)=$ $\lambda_{i}$, given the stimulus projection on the $d$ linear components, $\mathbf{X}_{i j}$, with $1 \leq j \leq d$.

The logistic regression makes no assumption about the distribution of the independent variables: they do not have to be normally distributed, linearly related or to have equal variance within each group [6]. In logistic regression the relationship between the predictor and response variables is not a linear function, instead it uses the logit transformation of $\lambda_{i}$. The linear logistic model assumes that the log-odds are linear, while the additive logistic replaces each linear term by a more general functional of the form

$$
\ln \left(\frac{\lambda_{i}}{1-\lambda_{i}}\right)=\alpha+\sum_{j=1}^{d} f_{j}\left(\mathbf{X}_{i j}\right),
$$

where $d$ is the linear space dimension and each $f_{j}$ is a general function like a cubic spline or a polynomial.

The adjustment of the additive logistic model can be done by applying the local scoring algorithm, that starts to compute an initial guess of $\lambda_{i}$, which is obtained by initializing $\hat{f}_{j} \equiv 0, \forall j$ and $\hat{\alpha}$ as

$$
\hat{\alpha}=\ln \left(\frac{\bar{\rho}}{1-\bar{\rho}}\right),
$$

where $\bar{\rho}$ is the spike count average over time. Then, the regression target variable $\mathbf{Y}_{i}$ is written as

$$
\mathbf{Y}_{i}=\hat{\alpha}+\sum_{j=1}^{d} \hat{f}_{j}\left(\mathbf{X}_{i j}\right)+\frac{\left(\rho_{i}-\lambda_{i}\right)}{\lambda_{i}\left(1-\lambda_{i}\right)}
$$

Constructing the regression weights $w_{i}=\lambda_{i}\left(1-\lambda_{i}\right)$ and fitting the additive model to the targets $\mathbf{Y}_{i}$ with a weighted backfitting algorithm, new estimates are achieved for $\hat{\alpha}, \hat{f}_{j}, \forall j$. An iterative procedure for the additive logistic regression is achieved [7] by computing equation 3 with the new estimated values, until the functions changes falls bellow a threshold.

The weighted backfitting algorithm used in the local scoring algorithm, is a general algorithm that can fit an additive model using any regression-type fitting mechanisms that supports weighting. It defines the $j$ th set of partial residuals as

$$
\mathbf{R}_{j}=\mathbf{Y}-\hat{\alpha}-\sum_{k \neq j} \hat{f}_{k}\left(\mathbf{X}_{k}\right)
$$

which provides a way for estimating each function $f_{j}$ given an estimation for all the others:

$$
f_{j} \leftarrow S_{j}\left[\mathbf{R}_{j} \mid \mathbf{X}, \mathbf{w}\right]
$$

where $S_{j}$ is the fitting operator. This backfitting procedure is analogous to a multiple regression for linear models. For more details about these algorithms and to generalized additive models theory refer to [6].

\section{MODELLING THE RETINA}

After stimulating a spiking neuron with a sequence drawn randomly from an ensemble, one can analyze the stimulus subsets that elicit spikes. Spike-triggered analysis exploits the fact that the spike-triggered stimulus ensemble can provide information about the neuron response characteristics. This method has been successfully exploited in several retina models [1] [5].

By considering $\mathbf{s}$ the raw stimulus ensemble, $\mathbf{s}_{s p k}$ is defined as the subset of stimulus that triggered a spike. A simple operation to do over these blocks of stimuli is the spiketriggered average (STA) which corresponds to the mean stimulus that elicit a spike:

$$
\mathbf{m}=\frac{1}{N} \sum_{i=1}^{N} \mathbf{s}_{s p k}
$$

It was shown that $\mathbf{m}$ is an unbiased estimation of the linear component of the LNP model as long as the stimulus distribution is spherically symmetric [1].

However, a single linear filter will fail to describe complex responses, and so the generalized version of the LNP model is introduced. It includes a nonlinear combination of several linear responses instead of just one. To recover this set of linear filters, more operations over $\mathbf{s}_{s p k}$ must be performed, such as the $2^{\text {nd }}$ order moment (covariance), which is the natural evolution of the $1^{\text {st }}$ order one (mean). The covariance matrix $\mathbf{C}$ is defined as:

$$
\mathbf{C}=\frac{1}{N-1} \sum_{i=1}^{N}\left(\mathbf{s}_{s p k}-\mathbf{m}\right) \cdot\left(\mathbf{s}_{s p k}-\mathbf{m}\right)^{\top}
$$

In order to identify the most significant kernels, a principal component analysis (PCA) is performed, looking for the directions that suffered a significative change from the raw stimulus ensemble to the spike triggered ensemble. Applying this technique, known as spike-triggered covariance, it is possible to identify a set of orthogonal (eigen-)vectors that correspond to: $i$ ) excitatory kernels, if the eigenvalues of the spike triggered ensemble increases relatively to the raw stimulus ensemble; and ii) suppressive kernels if it decreases, defining a multi-dimensional linear stage [5].

Now all projections of the stimuli on each linear component must be combined to obtain the probability of a spike being generated at a given time instant. This is where the particular use of the generalized additive models is introduced with the additive logistic regression: the log-odds of a spike being elicited is given by the sum of the nonlinear transformation of the linear projections. In particular, the nonlinear functions $f_{j}$ used in this paper are polynomial functions with the form

$$
f_{j}(z)=\sum_{c=1}^{Q} \beta_{c} \cdot z^{c}
$$

where the coefficients $\beta$ of the polynomial of degree $Q$ are adjusted in a weighted least squares sense. The zero order co- 
efficients of each polynomial are imposed to be zero because $\alpha$ in equation 1 can take the constant terms into account.

The model for spike generation is an inhomogeneous Poisson process, that assumes that the firing probability at any instant of time depends only on the stimulus itself and not on the past spikes. This means that the Poisson process rate is simply $\lambda_{i}$, the probability of having a spike at time instant $i$.

The assumption that each spike is generated independently is coarse, as the refractoriness of the neuron affects the spike generation. Thus, a new term $r_{i}$ was included to model the refractory period, which depends on the elapsed time since the last spike occurred, such that the Poisson process rate becomes $r_{i} \cdot \lambda_{i}$. The refractory term models the 3 states of the neuron: (1) the refractory period which disables the cell from generating any spikes $\left(r_{i} \simeq 0\right),(2)$ the recovery from the refractory period, where their ability to generate new spikes increases with the time elapsed since last spike ( $r_{i}$ rises towards 1 ), and (3) the neuron regular state, where the probability of generate a spike only depends on $\lambda_{i}\left(r_{i} \simeq 1\right)$. For more details about spike generation mechanisms and how to model the refractory term refer to [8].

\section{RESULTS}

The methods and algorithms proposed were implemented and tested with the experimental data used in the paper [9], consisting of 12 trials of full field white noise stimulation for a salamander ON cell, where each trial has a duration of 10 seconds with an average count of 8.34 spikes per second. The data was split for training (60\%) and testing (40\%) purposes.

To compare two spike trains, the spike time metric proposed in [10] is used $d_{q}^{\text {time }}$. This metric measures the distance between the spike trains $\rho_{a}$ and $\rho_{b}$ as the minimum cost to transform the spike train $\rho_{a}$ into $\rho_{b}$ by the successive application of a set of allowed elementary operations, namely: insertion, or deletion, of an individual spike, both with unitary cost per spike; and the time shift in the occurrence of a spike, with a cost of $q$ per time unit, resulting in cost of $q|\Delta t|$ for a time shift of $\Delta t$. In order to be comparable, the error values are normalized by the spike train length.

The spike time metric evaluates the complete model, including the spike generator, but since we are mainly focused on the nonlinear block, its output must be evaluated too. For that purpose, the normalized mean squared error (NMSE) was used, which is defined as [8]

$$
\mathrm{NMSE}=\frac{\sum_{i=1}^{N}\left(\lambda_{i}-\hat{\lambda}_{i}\right)^{2}}{\sum_{i=1}^{N}\left(\lambda_{i}-\langle\lambda\rangle\right)^{2}}
$$

The NMSE was computed for the estimated and observed firing rates. The last can be extracted from the PeriStimulus Time Histogram (PSTH) computed across several trials of the same experiment [1].

The model performance was analyzed while varying the number of linear components $d$ and the polynomials order $Q$. From the results in fig. 2, it can be observed that high order polynomials are unnecessary, and in fact, they just lead to
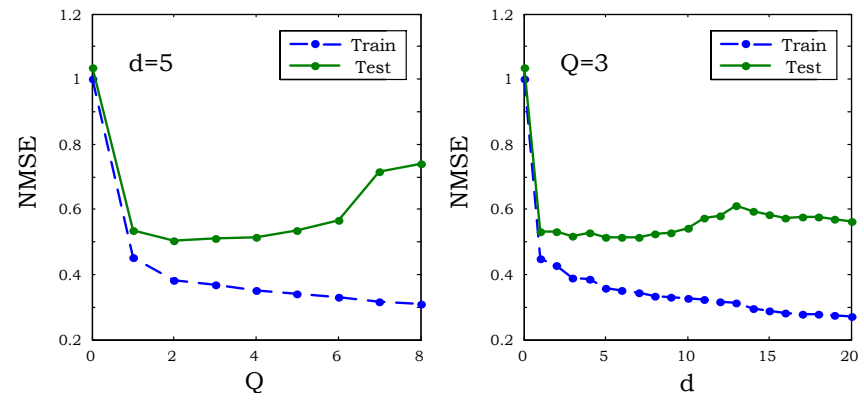

Fig. 2. Model evaluation with NMSE of train and test data sets, varying the polynomials order $Q$ and number of linear components $d$
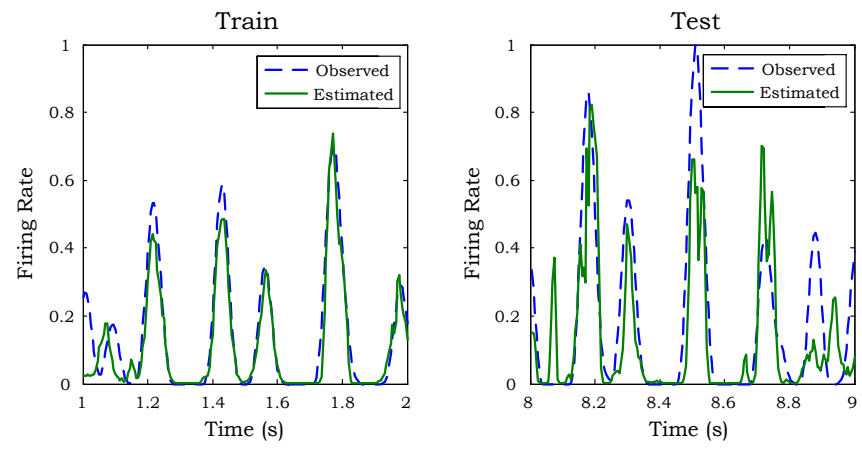

Fig. 3. Comparison between the estimated and the observed firing rate $\lambda_{i}$, for the train and test data sets $(d=5, Q=3)$

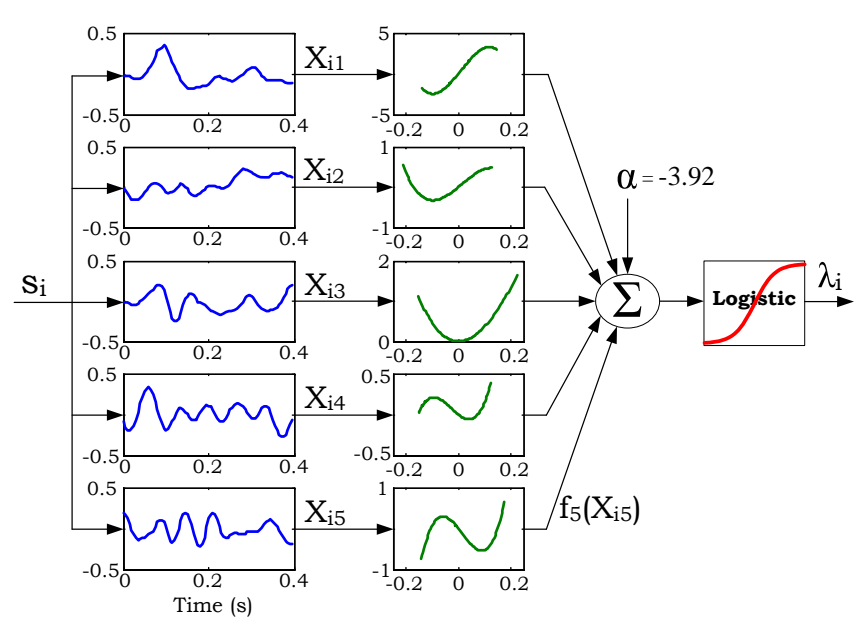

Fig. 4. Additive Logistic model: The linear components of the model are depicted along with the estimated nonlinear functions 
overfit since the NMSE of the test data starts to increase from $Q=2$. Holding the polynomials order, and varying the number of linear components, the model performance has been also analyzed. The first linear component, given by the STA in equation 6 , is the one that offers the most relevant information, while in this case, the remaining linear components have a small contribution to the train and test NMSE.

From the analysis above, a pair of $Q$ and $d$ was chosen and the estimated firing rate was compared with the observed one, in fig. 3. A polynomials order $Q=3$ was applied and the number of linear components was $d=5$. Fig. 4 illustrates the obtained additive logistic model, where both the linear and nonlinear components are depicted. It provides information about the contribution of each linear component, giving some insight about the retina behavior when stimulated.

Linking the firing rate estimate to the Poisson spike generator, a set of spike trains was generated. Using the spike time metric with $q=50 / s$, the spike trains were compared, within each set of trials and cross-comparing them. The results in table 1 resumes the model evaluation for the parameters chosen, where the good relationship between the train and test errors is numerically demonstrated. A framework to evaluate different retina models [11] is now being developed to relatively assess the obtained results. Finally, the estimated and observed spike trains are depicted in fig. 5 .

\begin{tabular}{|c|c|c|c|}
\hline \multicolumn{2}{|c|}{} & Train Data & Test Data \\
\hline \multirow{2}{*}{$d_{q=50 s^{-1}}^{\text {Nime }}$} & Observed trials & 0.389 & 0.561 \\
& Estimated trials & 68.48 & 36.55 \\
& Cross-trial & 75.32 & 71.96 \\
& &
\end{tabular}

Table 1. Error measures

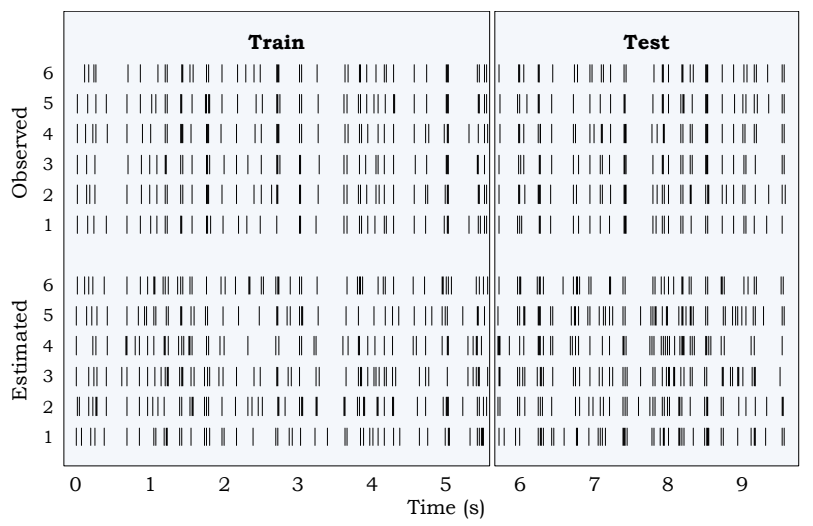

Fig. 5. Observed and estimated spike trains

\section{CONCLUSIONS}

We demonstrated how generalized additive models can be used to model the retina by following a probabilistic point of view of the retina neural code. In particular, additive logistic regression was used to estimate the nonlinear functional of the well known LNP model, proving to be a flexible statistical method to identify and characterize the nonlinear effects existent in the neural code.

The proposed model provided a way to combine several stimulus features, in a nonlinear form. It is easily extensible to accommodate more severe nonlinearities just by changing the number of linear components. The number of parameters and the data set size needed to successfully do it, grow linearly with the number of linear components, while still retaining much of its interpretability.

\section{REFERENCES}

[1] E.J. Chichilnisky, "A simple white noise analysis of neuronal light responses," Network: Computation in Neural Systems, vol. 12, no. 2, pp. 199-213, 2001.

[2] J.W. Pillow and E.P. Simoncelli, "Dimensionality reduction in neural models: An information-theoretic generalization of spike-triggered average and covariance analysis," Journal of Vision, vol. 6, no. 4, pp. 414-428, 2006.

[3] L. Paninski, J.W. Pillow, and E.P. Simoncelli, "Maximum Likelihood Estimation of a Stochastic Integrateand-Fire Neural Encoding Model," Neural Computation, vol. 16, no. 12, pp. 2533-2561, 2006.

[4] H. Kolb, "How the retina works," American Scientist, vol. 91, no. 1, pp. 28-35, 2003.

[5] O. Schwartz, E.J. Chichilnisky, and E.P. Simoncelli, "Characterizing neural gain control using spiketriggered covariance," Adv. Neural Information Processing Systems (NIPS* 01), vol. 14, pp. 269-276, 2002.

[6] T. Hastie, R. Tibshirani, J. Friedman, et al., The elements of statistical learning: data mining, inference, and prediction, Springer, 2001.

[7] T.J. Hastie, Generalized Additive Models, Chapman \& Hall/CRC, 1990.

[8] M.J. Berry and M. Meister, "Refractoriness and Neural Precision," Journal of Neuroscience, vol. 18, no. 6, pp. 2200-2211, 1998.

[9] J. Keat, P. Reinagel, R.C. Reid, and M. Meister, "Predicting Every Spike A Model for the Responses of Visual Neurons," Neuron, vol. 30, no. 3, pp. 803-817, 2001.

[10] J.D. Victor and K.P. Purpura, "Metric-space analysis of spike trains: theory, algorithms and application," Network: Computation in Neural Systems, vol. 8, no. 2, pp. 127-164, 1997.

[11] J.C. Martins and L.A. Sousa, "Performance comparison of computational retina models," International Conference on Visualization, Imaging and Image Processing, pp. 156-161, 2005. 